Revue d'Études Françaises

$\mathrm{N}^{\mathrm{o}} 24(2020)$

DOI : $10.37587 /$ ref.2020.1.13

\title{
ADRIENN GULYÁS
}

\section{Traduire la transgression verbale}

The article focuses on the translation of verbal transgression, the problems it raises for the translator and strategies that can be used to solve them. Since verbal transgression is intricately linked to its immediate social context, it is extremely hard to transpose to another cultural and linguistic environment. On the other hand, the presence of verbal violence in a literary text is a very strong marker, both stylistically and dramaturgically, so it cannot be ignored in translation. Examples to illustrate the translator's dilemmas are taken from Morgan Sportès' social documentary novel, Tout, tout de suite (2011) and its Hungarian translation by Ildikó Lörinszky (2014).

\section{Qu'est-ce que la transgression verbale?}

La transgression verbale fait partie des actes de langage et, en tant que tel, elle trouve sens à travers la réalité socio-culturelle où elle est utilisée. Du fait de sa visée et de ses connotations métalinguistiques, son décodage présuppose la connaissance intime du contexte socio-culturel où elle a lieu (cf. Fischer, 2004). Dans les jurons, les insultes ou les grossièretés, "le sentiment de vulgarité, d'agressivité ou de grossièreté repose souvent sur des formes choquantes et décalées par rapport aux codes sociaux attendus » (Moïse, 2011 : 29 ; nous soulignons). Ce décalage par rapport à la norme, sociale ou linguistique, constitue l'essence même de la transgression, car c'est par antagonisme à la norme qu'elle se définit.

Les actes de langage transgressifs s'accompagnent également d'une décharge d'émotion. Ils peuvent avoir une fonction interjective comme les jurons et les grossièretés ou une fonction appellative comme l'insulte (Lecomte, 1999: 179-180). L'émotion qui se verbalise par ces actes est souvent symptomatique d'un mal-être psychologique et/ou social. Elle peut donner voix à des sentiments d'injustice, de non-reconnaissance ou de frustration d'un individu ou d'un groupe social (voir aussi à ce sujet Moïse, 2011: 33 ou Goudaillier, $2001: 6-10)$. La transgression verbale peut également marquer des 
rapports de pouvoir ou de connivence au sein d'un groupe ou signaler l'appartenance à un groupe social particulier.

La transgression verbale qui marque l'appartenance tout en se démarquant de la norme acquiert une importance particulière dans les variétés périphériques de la langue, notamment dans le parler des cités. On va voir à travers les exemples pris dans le roman de Sportès Tout, tout de suite comment la trangression verbale sera à la fois porteuse de l'appartenance à un groupe, celui des jeunes de banlieue, et de la rancune, voire de la haine contre tous ceux qui semblent représenter, du moins à leurs yeux, la norme sociale. Selon Goudaillier (2001: 9), « [1]a violence verbale ou l'impression ressentie d'une telle violence est une des manifestations les plus perceptibles des fractures linguistique et sociale qui s'opèrent » dans les banlieues. Dans ce qui suit, nous examinerons quels rôles et effets la transgression verbale peut avoir dans les œuvres littéraires.

\section{La transgression verbale dans les œuvres littéraires et les difficultés de sa traduction}

La présence de la transgression verbale dans les œuvres littéraires n'est jamais aléatoire : loin de la spontanéité et du caractère irréfléchi qu'elle peut avoir dans une interaction orale, la violence linguistique est un acte réfléchi et bien calculé en littérature. L'écrivain qui construit un texte littéraire sait pertinemment quelle place il veut accorder à la violence verbale dans son texte. Par les propos violents, l'écrivain peut caractériser un personnage comme c'est le cas du chef du gang des barbares dans le roman de Sportès. La violence verbale peut aussi remplir une fonction dramaturgique et présager un acte violent.

Si l'auteur construit le texte, le traducteur, à son tour, le déconstruit. Dans un premier temps, le traducteur retrace le travail de l'auteur et essaie de déceler ses intentions. Il détermine notamment la fonction de la violence verbale dans l'œuvre et évalue le sens, le contexte et surtout l'effet que la transgression verbale peut avoir dans la culture-source. La traduction des transgressions verbales diverses requiert ainsi une connaissance profonde de la culture dans laquelle le texte-source a été écrit et exige « une analyse préalable des effets connotatifs à traduire » (Ladmiral, 1994: 156). Néanmoins, même après analyse des effets connotatifs, le traducteur manque souvent de prise sur le sens littéral ou le contexte social, impossibles à rendre puisqu'ils n'ont pas 
d'équivalents dans la culture-cible. Il lui reste donc l'effet et la fonction de cet effet dans le récit comme points de départ pour la traduction.

Le meilleur moyen de reproduire la violence verbale dans un autre contexte linguistique et social est probablement d'analyser et de reproduire son effet plutôt que de s'efforcer de lui trouver un équivalent sémantique qui n'aura forcément pas les mêmes connotations, et ne fera pas le même effet, ou pire, ne fera pas d'effet du tout. Les pertes de connotations, de références métalinguistiques dues à la différence de la culture-source et de la culture-cible sont malheureusement inévitables, ce qui est certainement frustrant pour le traducteur, mais passent souvent inaperçues pour les lecteurs de la traduction.

\section{La traduction de la violence verbale dans Tout, tout de suite}

Les exemples pour illustrer la traduction de la transgression verbale sont tirés du roman-enquête de Morgan Sportès, intitulé Tout, tout de suite, qui porte sur l'affaire du gang des barbares. Ce roman documentaire raconte l'enlèvement, la séquestration et l'assassinat d'un jeune commerçant juif, Ilan Halimi par un groupe de jeunes de banlieue, menés par Youssouf Fofana, en 2006. Le roman de Sportès, minutieusement documenté, mêle un style documentaire, journalistique avec des dialogues imitant la langue des cités, le parler des «cailleras». La violence verbale est surtout présente dans les énoncés de l'auteur du crime, Fofana, alias Yacef.

Avant de passer à la discussion de la violence verbale, il nous paraît important de commenter la traduction du terme caillera. Bien que ce mot ne puisse pas être qualifié de transgressif, il représente bien la fracture linguistique et sociale, caractéristique des cités en France, pour reprendre l'expression de Goudaillier (2001: 9). Caillera est la forme verlanisée de racaille, et garde toujours les connotations péjoratives de son étymon. Néanmoins, le terme se mue par la verlanisation et s'emploie également par les jeunes des cités pour se désigner eux-mêmes, il a donc une fonction identitaire. Caillera représente à la fois la langue cryptique, mais ingénieuse des jeunes de banlieue et leur réalité sociale caractérisée par le chômage, la violence, le manque de perspectives ainsi que le mépris social dont ils sont l'objet.

Caillera pose problème au traducteur, d'abord, parce que le monde des cités avec son métissage culturel et linguistique, ses problèmes sociaux liés à l'immigration ne correspond à aucune réalité socio-culturelle hongroise. De plus, le verlan n'existe pas en tant que procédé de création lexicale en hongrois, 
il est pourtant une des spécificités de la langue des cités. Il faut préciser d'emblée que le transfert de caillera dans une autre culture et langue sans aucune perte de connotations est impossible. Caillera apparait sept fois dans le texte, souvent pour décrire l'accent de Yacef sans le nommer. Sa traduction reste cohérente tout au long: kültelek/kültelki «banlieue/banlieusard», variante un peu vieillie et choisie de sa variante neutre külváros/külvárosi, utilisée seulement une fois :

- Mettez-le par terre, ce fils de pute! lance une voix rauque, grave, avec l'accent caillera. S 66

- Teperjétek le ezt a rohadt gecit! - kiáltja egy rekedtes, mély hang, kültelki akcentussal. L 62

- Si tu paies pas, dit une voix de caillera à l'autre bout du fil, j'te dénonce à l'ordre des médecins pour escroquerie ! Tu m'écoutes, pédé ? S 42

- Ha nem fizetsz - szólal meg a vonal másik végén külvárosi kiejtéssel egy

hang -, feljelentelek csalásért az orvosi kamaránál! Figyelsz rám, te geci? L 40 ${ }^{1}$

L'expression kültelek sonne énigmatique et lointaine en hongrois. En fin de compte, on a du mal à imaginer ce que c'est exactement. On peut défendre le choix de ce terme en disant qu'il ne fait qu'augmenter l'effet d'étrangéité qui, bien qu'à des degrés divers, est le propre de toutes les traductions. Cette étrangéité rappelle aux lecteurs hongrois qu'il s'agit là d'un phénomène propre à la culture française. Il faut admettre cependant que caillera, avec toutes ses connotations culturelles implicites qui n'existent pas dans la culture-cible, est impossible à traduire et, de ce point de vue-là, il résume bien les dilemmes du traducteur pour transposer la langue des cités dans une autre culture.

L'emploi de la transgression verbale paraît avoir un double objectif chez Sportès. Comme nous l'avons mentionné plus haut, l'écrivain utilise la transgression verbale surtout dans les énoncés de Yacef: elle caractérise le personnage le plus agressif et cruel du gang des barbares. D'autre part, les insultes ou injures proférées par Yacef font partie intégrante de ses chantages et menaces de mort. La violence de ses paroles offre un parallèle à la violence de ses actes et son personnage de brute sociopathe. Cette violence dans les propos de Yacef présage aussi le dénouement tragique du récit. La brutalité des propos

\footnotetext{
${ }^{1}$ Dans ce qui suit, les exemples tirés de l'original seront signalés par l'initiale de l'écrivain, suivie du numéro de la page du roman en français. Les exemples tirés de la traduction hongroise seront signalés par l'initiale de la traductrice, suivie du numéro de la page de la traduction.
} 
de Yacef sert à caractériser le personnage, maintenir le suspens, et laisse deviner la fin de l'histoire, donc elle a également une fonction dramaturgique.

Du flot des putains et des merdes, termes d'injure largement utilisés en français comme en langue des cités, on relève ici la putain de ta race qui est, en revanche, marqué « parler des cités »:

La putain de ta race si tu causes, t'entends? menace-t-il. Si tu causes ta darone, je la plante, t'entends, j'l'égorge, et toi aussi je te... te... te... tue. Et ton frère pareil! Ta sœur... S 45

- Az egész fajtádat kiirtom, ha pofázol, értve vagyok? - mondja neki fenyegetően. - Ha pofázol, megkéselem az anyádat, érted, megfojtom, és téged is, tttttéged is mmmegöllek. És az öcsédet is! Meg a nővéredet. L 42-43

Avec cette injure et la menace qui suit, les deux s'attaquant aux tabous de la race, la figure de la mère et la sainteté de la famille, on devine l'appartenance religieuse et la classe sociale du locuteur. Yacef-Fofana, d'origine ivoirienne, est de fait musulman pratiquant, habitant de la banlieue parisienne. La version hongroise transforme ingénieusement l'injure en menace : «Je vais éradiquer ton espèce ». Aussi, la traductrice préserve-t-elle l'idée de l'attaque au couteau (megkéselem), mais transforme l'égorgement, à très fort effet connotatif, qu'elle aurait pu garder, en étranglement (megfojtom). On y perd la connotation «terrorisme islamiste », l'égorgement d'otages étant un sévice régulièrement mis en scène dans les vidéos de propagande islamiste type Al-Qaïda. Les implications des tabous évoqués et de l'injure sont autant de marqueurs linguistiques et culturels qui se perdent en traduction. Ce que la traduction garde de ce passage, en revanche, c'est sa violence extrême, non moins importante du point de vue narratif.

Dans ses insultes qui accompagnent ses chantages et menaces, Yacef traite souvent ses interlocuteurs des synonymes d'homosexuel pour les rabaisser et anéantir (par exemple, le père du jeune homme séquestré) : cette tapette de Zacharie (S 88), pédé (S 42, 351, 376), petit pédé (S 89, 231, 251), enculé ( $\mathrm{S}$ $88,272,345)$, espèce de pédé (S 88). Le contexte social des cités, très patriarcal et machiste, ne fait que renforcer le caractère homophobe de ces insultes. En effet, de nombreux articles de journaux évoquent même actuellement le drame quotidien des homosexuels des cités, ostracisés, agressés, humiliés par leur entourage immédiat, souvent par leur famille. Être traité de " pédé» en parler des cités est l'une des insultes suprêmes. Ce contexte social particulier qui renforce l'intensité des insultes à caractère homophobe est un autre exemple 
des références culturelles, métalinguistiques qui se perdront inévitablement en traduction.

Comme équivalent des insultes à caractère homophobe, on trouve onze occurrences de köcsög/kisköcsög « enculé/petit enculé », quatre occurrences de buzi "pédé » et une de buzeráns "pédéraste ». Köcsög/kisköcsög, termes les plus utilisés par la traductrice, signifient littéralement « pot/petit pot», mais ont acquis le sens argotique de «homosexuel passif». Ils s'utilisent aussi, de plus en plus, pour désigner une personne méprisable, dénuée de tout principe moral, synonyme de «salopard». Dans ce sens, köcsög est interchangeable avec geci (voir plus bas). Köcsög possède également un emploi adjectival où il ajoute un sens péjoratif au substantif qu'il précède ( $a z$ a köcsög Yacef L 378 pour traduire ce fils de pute de Yacef S 368). Malgré la fréquence de son usage, cette insulte garde toujours sa connotation homophobe et vulgaire. Le mot buzi " pédé » apparaît deux fois dans des dialogues (L 88, 264), une fois dans la narration (buzi cowboyok L 158, comme équivalent de cow-boys pédés S 166), une fois en emploi adjectival (L 342). Buzeráns " pédéraste » (L 85) n'a qu'une seule occurrence en tant qu'équivalent de pédé dans l'original.

Geci, variante triviale de "sperme», signifiant également "personne méprisable », a cinq occurrences dans la traduction hongroise. Il a également un emploi adjectival et s'utilise dans le parler des jeunes en tant que marqueur de discours, ce qui indique un processus de désémantisation du terme. La traductrice l'utilise parfois comme l'équivalent de pédé en français. La connotation homophobe n'y est pas, mais le terme est très insultant et grossier :

- Tu m'écoutes, pédé ? S 42

- Figyelsz rám, te geci? L 40

- Enculé de flic, putain de ta mère, sale flic de merde ! S 32

- Szarfaszú zsaru, a kurva anyádat, rohadt geci! L 30

Emploi adjectival :

Espèce de pédé, enculé ! 58

Te rohadt geci patkány! L 85

Dans ce dernier exemple, geci modifie le substantif patkány «rat », employé ici au sens de "personne méprisable». Comme on voit dans ces exemples, l'adjectif rohadt "pourri » apparaît à deux reprises dans les insultes. Cet adjectif forme des expressions figées avec certains substantifs dans les insultes, ainsi son insertion dans le texte hongrois «domestique » la traduction. Même si 
ces expressions hongroises manquent certains effets connotatifs et références culturelles de l'original, elles sont considérées comme grossières et vulgaires, et le fait qu'elles apparaissent dans un texte littéraire ne fait que rehausser leur effet. La brutalité, le mépris et la rage de celui qui les profère sont évidents pour le lecteur.

Dans un courriel personnel, Ildikó Lőrinszky nous a fait part de son ennui à voir des mots de la langue des cités s'infiltrer dans la narration. Au début, le style du narrateur se sépare nettement du parler des cités. Vers la fin, le narrateur semble mêler les deux styles. Puisque les lecteurs hongrois ne connaissent pas l'original, la traductrice redoute qu'ils accuseront la traduction de brouiller les styles au départ bien distincts du narrateur et des jeunes des cités. Étant donné que la tension monte de façon considérable vers la fin du roman, cet emmêlement stylistique n'est vraisemblablement pas aussi gênant pour le lecteur que pour le traducteur.

Nous avons déjà évoqué l'exemple de buzi cowboyok L 158 (cow-boys pédés S 166) dans le texte narratif, mais voici deux exemples du mot bouffon relevés dans la narration et une occurrence dans un dialogue. Bouffon signifie «quelqu'un de complètement nul, connard», mais comme le remarque Goudaillier, il s'agit également d'une des «insultes suprêmes en parler des cités ». Bouffon désigne «tous ceux extérieurs à la cité, ceux qui ont de l'argent, des bourgeois » (2001: 72).

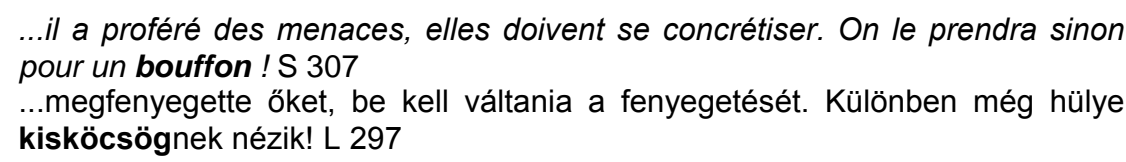

En inspectant son portable, Rapetou y a trouvé le nom d'un bouffon qu'il kiffe pas. S 376

Rapetou ellenőrizte a mobilját, és meglátta egy seggfej nevét, akinek nem bírja a pofáját. $L 366$

- Alors, t'es un marrant, toi ! lance-t-il à Gérard, t'es un bouffon ! Tu crois que tu vas te tirer comme ça ? S 246

- Na, mondhatom, jó firma vagy, te kisköcsög! - veti oda Gérard-nak. - Azt hiszed, csak úgy kiszállhatsz? L 238

L'emploi de bouffon dans le premier exemple peut être considéré comme du style indirect libre, mais dans le deuxième exemple, il s'agit clairement d'un dérapage stylistique de Sportès. Dans le troisième exemple, le mot est utilisé 
dans le sens citéen que lui confère Goudaillier: Krack s'énerve parce que Gérard ne veut plus garder l'otage. Bouffon est ici employé comme une insulte, synonyme de traître presque, et désigne quelqu'un qui vient de l'extérieur : Gérard habite une autre banlieue, et il est blanc. La traduction fait bien la différence entre les contextes où bouffon est employé comme insulte (exemples 1 et 3 ) et le contexte où il veut simplement dire «tocard» (exemple 2), en utilisant kisköcsög " petit pédé » et seggfej « connard », respectivement.

\section{Conclusion}

Selon la traductrice Ildikó Lőrinszky elle-même (cf. : courriel personnel mentionné plus haut), son plus grand dilemme n'était pas ce qu'elle avait pensé avant d'entamer le travail de traduction du roman de Sportès: il était relativement facile de trouver le sens des expressions du langage des cités sur Internet. Son dilemme consistait plutôt à leur trouver des équivalents hongrois. Le roman de Sportès décrit une réalité socio-culturelle bien française, l'existence des cités avec les problèmes sociaux qui les caractérisent, souvent inséparables des questions de race et de multiculturalisme. Cette réalité des cités, la langue et l'identité des «cailleras» sont inconnues des lecteurs hongrois et n'ont pas de phénomènes équivalents dans la société hongroise.

De ce point de vue, les pertes pour la traduction et la réception de l'œuvre en Hongrie sont inévitables. Certains tabous comme la race ou la figure de la mère, bien qu'ils existent en hongrois, ont des connotations différentes : en hongrois, leur usage est général, en français, ils sont connotés "parler des cités » et impliquent des origines culturelles nord-africaines ou méditerranéennes. Certains procédés linguistiques dont le parler des cités fait un usage extensif, tels le verlan (keuf, renoi, reubeu), des suffixes péjoratifs (pétasse), les emprunts à l'arabe (kiffer, wesh) sont également impossibles à reproduire en hongrois.

Ce que le traducteur peut faire pour compenser ces pertes de connotations propres à la culture-source, et ce que le duo d'Ildikó Lőrinszky et son éditeurargotologue Dávid Szabó a fait d'ailleurs avec brio, c'est de signaler la présence d'une transgression verbale dans le texte hongrois. Pour ce faire, la traductrice s'est inspirée de l'argot des prisons pour lequel bon nombre de ressources sont accessibles en ligne. De toute manière, la transmission du contenu exact, des équivalences littérales des transgressions verbales de l'original paraissent secondaires par rapport à l'effet qu'elles produisent. Si 
l'on considère le roman-enquête de Sportès dans son intégralité, il apparaît clairement que la transgression verbale va de pair avec la brutalité des événements narrés. Au lieu d'insister sur le mot à mot et les équivalences lexicales, la traductrice se concentre sur la fonction de la transgression verbale dans le texte et, en recréant un effet de choc, renforce la violence de l'histoire par la violence des mots.

\section{Corpus}

SPORTÈS Morgan (2011), Tout, tout de suite, Paris, Fayard.

SPORTÈS Morgan (2014), Mindent azonnal, Budapest, Park Könyvkiadó, trad. Ildikó Lörinszky.

\section{Bibliographie}

FISCHER Sophie (2004), «L'insulte : la parole et le geste », Langue française, $\mathrm{n}^{\mathrm{o}}$ 144/4, p. 49-58. DOI : 10.3917/lf.144.0049

GOUDAILLIER Jean-Pierre (2001), Comment tu tchatches!, Paris, Maisonneuve et Larose.

LADMIRAL Jean-René (1994), Traduire : théorèmes pour la traduction, Paris, Éditions Gallimard.

LECOMTE Marie-Christine (1999), «Injures, jurons et blasphèmes : des performatifs bien ancrés dans le réel », in : Ré-inventer le réel (Th. Dutoit, T. Harris éds.), Tours, Presses Universitaires François-Rabelais, Coll. « GRAAT 20 », p. 179-189. DOI : 10.4000/books.pufr.4136

MOÏSE Claudine (2011), «Gros mots et insultes des adolescents », La lettre de l'enfance et de l'adolescence, $\mathrm{n}^{\circ}$ 83-84(1), p. 29-36. DOI : 10.3917/lett.083.0029

\section{ADRIENN GULYÁS}

Université Károli Gáspár de Budapest

Courriel : gulyas.adrienn@kre.hu 